\title{
Outcome analysis of solitary osteochondromas of femur managed surgically: a series of 32 patients
}

\author{
Sanjeev Gupta, Abdul Ghani, Bias Dev, Rahul Mahajan, John Mohd, \\ Amarjeet Singh, Zubair A. Lone*
}

Department of Orthopaedics, Government Medical College, Jammu, India

Received: 20 September 2020

Revised: 03 November 2020

Accepted: 04 November 2020

*Correspondence:

Dr. Zubair A. Lone,

E-mail: lonezubair1255@gmail.com

Copyright: (C) the author(s), publisher and licensee Medip Academy. This is an open-access article distributed under the terms of the Creative Commons Attribution Non-Commercial License, which permits unrestricted non-commercial use, distribution, and reproduction in any medium, provided the original work is properly cited.

\section{ABSTRACT}

Background: Osteochondroma is the commonest benign bone tumor in humans. This tumor may either be solitary or present as multiple lesions. Solitary osteochondromas are much more common. Femur is the single most common long bone involved followed by tibia and humerus. Osteochondromas may be either asymptomatic or may present as pain, pressure symptoms or functional impairment. Most of the symptomatic lesions need surgical excision.

Methods: This was a prospective study which included 32 patients with solitary osteochondromas of femur, who were managed with surgical excision. Only the symptomatic cases were operated. Minimum follow-up period was 18 months after surgery.

Results: This study involved 23 males (71.87\%) and nine females (28.12\%). 30 osteochondromas (93.75\%) were located in the distal femoral metaphysis while as only two cases $(6.25 \%)$ of proximal metaphyseal involvement were seen. The commonest indication for surgery was pain. Other indications included cosmetic causes, restricted joint movement, fracture of osteochondroma and paresthesia or numbness. The complications included post-operative hematoma formation which was seen in 2 patients $(6.25 \%)$, infection which was seen in one patient $(3.12 \%)$, postoperative hypoaesthesia in 2 patients $(6.25 \%)$ and recurrence of the tumor occurred in one patient $(3.12 \%)$.

Conclusions: Surgical excision of the solitary symptomatic femoral osteochondromas is a rewarding and safe procedure with minimal complications.

Keywords: Osteochondroma, Femur, Extra-periosteal excision, Benign bone tumor

\section{INTRODUCTION}

World Health Organization (WHO) defines osteochondromas as bony projections, covered by a cartilaginous cap, which lie at the outer surface of the bone. ${ }^{1}$ Osteochondroma is the most common benign bone tumor in humans and constitutes around $35 \%$ of all benign bone tumors. ${ }^{2}$ It was believed that osteochondroma is not a true neoplasm but a growth aberration that occurs due to ectopic growth plate. However recent literature suggests that the osteochondromas are truly neoplastic in origin. ${ }^{3}$ The tumor predominantly contains bony tissue; however, it is the cartilaginous cap which is responsible for its growth. ${ }^{4}$ Osteochondromas are usually seen in children and young adolescents and the growth typically ceases as the skeletal maturity is reached. Osteochondromas commonly present as a solitary lesion and rarely as multiple lesions. Solitary osteochondromas comprise of around $90 \%$ of the cases and most of the patients with multiple lesions have multiple hereditary osteochondromatosis. $^{5}$ Most of the solitary osteochondromas arise due to separation of a nest of cartilage cells from the physis during development which retains its growth potential; however, trauma and irradiation have also been reported in literature as causes. ${ }^{6}$ 
Some reports suggest osteochondromas to have a slight male preponderance., ${ }^{4,7}$ The femur is involved by osteochondromas in around $30 \%$ cases; hence, it is the single most common bone involved. ${ }^{7}$ The commonly involved long bones are the femur, tibia and humerus. ${ }^{8}$ Involvement of the knee joint is observed in around $40 \%$ of the patients. ${ }^{9}$ The region of the bone involved is metaphysis in almost all the cases and the tumor tends to grow away from the joint. ${ }^{6}$ Most of the osteochondromas are asymptomatic and may be observed as incidental findings on clinical examination and radiographs. ${ }^{10}$ The symptomatic cases may present due to pain, which is either due to indentation of the tumor into the soft tissues or a fractured osteochondroma. ${ }^{11}$ Few may present as a painless swelling located at the ends of the long bones, which slowly increases in size and sometimes rapidly during the growth spurt. ${ }^{6}$

Sometimes this swelling may even press upon important neurovascular structures. Clinical examination and plain radiographs are usually enough to clinch the diagnosis of an osteochondroma in long bones. ${ }^{6}$ However, advanced imaging studies like computer tomography (CT) and magnetic resonance imaging (MRI) may be performed to determine the relationship of the tumor with the adjacent soft tissue structures and to evaluate the cartilaginous cap. ${ }^{12}$ Not every solitary osteochondroma needs to be dealt surgically. ${ }^{13}$ Patients with solitary lesions who are asymptomatic can be managed conservatively but continuous follow-up is advisable. ${ }^{13}$ Surgical excision of the tumor is the mainstay of the treatment in symptomatic cases. $^{4}$

This study aimed at studying the outcome of the solitary osteochondromas of the femur treated with surgical excision.

\section{METHODS}

This was a prospective study, which included 32 patients with solitary osteochondromas of femur managed surgically, from July 2014 to October 2019 in the department of orthopaedics, Government Medical College, Jammu. A detailed history was taken and thorough clinical examination was done in all patients. Radiographs were taken in all cases to confirm the clinal diagnosis. CT and MRI were performed in several cases to know about the size of the cartilaginous cap and relationship with adjacent neurovascular structures. An informed written consent was taken from all the patients included in the study. The complications of the procedure were fully explained to the patients and all the patients agreed to take part in the study.

\section{Inclusion criteria}

The study included patients with solitary osteochondromas, osteochondromas of femur, patients of any age group and any sex.

\section{Exclusion criteria}

The study excluded patients with multiple osteochondromas, osteochondromas of other bones except femur, associated primary malignant tumors of bone, nonconsenters for surgery, and morbid patients unfit for surgery.

The surgery was performed under spinal anaesthesia in all cases. The choice of the approach was made depending upon the easier accessibility to the tumor. The osteochondromas located in the distal femoral metaphysis were removed using medial or lateral approaches depending upon the location of the tumor. Osteochondromas located in the proximal femur were removed using posterior approach. Extra-periosteal excision of the tumor was performed using various osteotomes. Adequate care of the adjacent soft tissue structures was taken and it was made sure that the tumor is completely removed. Hemostasis was achieved to avoid post-operative hematoma formation. A suction drain was placed in all cases. Soft tissue was closed back in layers. The tumor was sent for histopathology in all cases to rule out any malignant changes. Patients were given injectable antibiotic doses before and after surgery. The intravenous antibiotics were continued for three days after surgery, according to the hospital antibiotic protocol. Antiseptic dressings were done at 48 hours post-surgery and the drain was removed at the same time. Skin sutures were removed at two weeks after surgery. Post-operative radiographs were taken to assess the adequacy of the excision. Most of the patients were discharged at third post-operative day. The patients were followed at 2 weeks, 6 weeks, 3 months, 6 months and 18 months. At every follow-up the patients were assessed for any complications. No patient was lost to follow-up and none of the patients died till the completion of this study. The statistical analysis of the data was carried out by using percentage, mean, range and standard deviation (SD).

\section{RESULTS}

The characteristics of the study participants have been summarized in Table 1. This study included 32 patients with solitary osteochondromas of femur, out of which 21 osteochondromas $(65.62 \%)$ were pedunculated and 11 osteochondromas $(34.37 \%)$ were sessile. All the osteochondromas were located in the metaphysis of the femur and no diaphyseal lesion was observed. 30 osteochondromas $(93.75 \%)$ were located in the distal femoral metaphysis (Figure 1) while as only two cases $(6.25 \%)$ of proximal metaphyseal (Figure 2) involvement were seen. This study involved 23 males $(71.87 \%)$ and nine females $(28.12 \%)$. A male to female ratio of $2.5: 1$ was observed. The mean age of the patients at surgery in this study was 17.5 years (range 11-28 years). The commonest indication for surgery was pain. Other indications included cosmetic cause, restricted joint motion, fracture of osteochondroma and paresthesia or numbness. None of the patients with vascular compression or malignant 
transformation were observed. Few complications were encountered in this study. Two patients $(6.25 \%)$ developed post-operative hematoma which resolved with serial aspiration and compression bandaging. Both of these patients had giant osteochondromas. Infection occurred in one patient $(3.12 \%)$ which was managed with serial debribements and antibiotics. Two patients (6.25\%) developed post-operative hypoaesthesia. Recurrence was seen in one patient $(3.12 \%)$ which occurred four years after the primary excision and was managed with re-surgery.

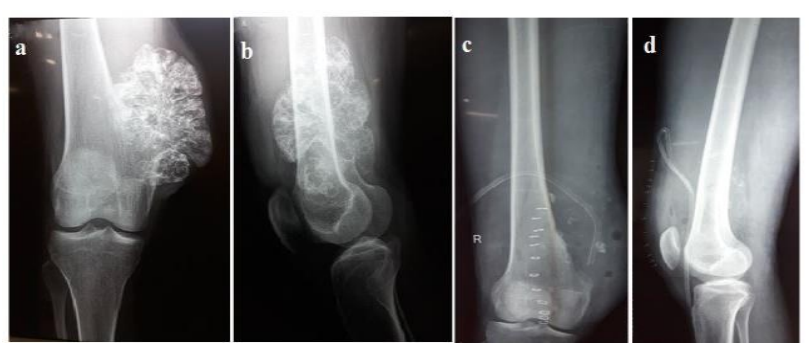

Figure 1: Radiograph (a) and (b) a sessile osteochondroma of distal femur in a 21 year old male who presented with dull aching pain and a huge swelling over medial aspect of right distal thigh, (c) and (d) surgical excision was done using a medial approach. Radiograph taken post-operatively showing complete removal of osteochondroma.

Table 1: Characteristics of the study participants $(n=32)$.

\begin{tabular}{|ll|}
\hline Characteristics & No. of patients (\%) \\
\hline Type of lesion & $21(65.62)$ \\
\hline Pedunculated & $11(34.37)$ \\
\hline Sessile & \\
\hline Location of tumor & $30(93.75)$ \\
\hline Distal femoral metaphysis & $02(06.25)$ \\
\hline Proximal femoral metaphysis & $0(0)$ \\
\hline Diaphysis & \\
\hline Gender distribution & $23(71.87)$ \\
\hline Males & $09(28.12)$ \\
\hline Females & $2.5: 1$ \\
\hline Male:female & $17.5 \pm 4.56($ range $11-$ \\
\hline $\begin{array}{l}\text { Age at surgery (years), } \\
\text { mean } \pm \text { SD (range) }\end{array}$ & $28)$ \\
\hline Indications for surgery & \\
\hline Pain & $18(56.25)$ \\
\hline Cosmetic & $05(15.62)$ \\
\hline Fracture of osteochondroma & $04(12.50)$ \\
\hline Restricted joint motion & $03(09.37)$ \\
\hline Paresthesia/numbness & $02(06.25)$ \\
\hline Complications & $02(06.25)$ \\
\hline Post-operative hematoma & $01(03.12)$ \\
\hline Infection & $02(06.25)$ \\
\hline Post-operative hypoaesthesia & $01(03.12)$ \\
\hline Recurrence & \\
\hline & \\
\hline
\end{tabular}
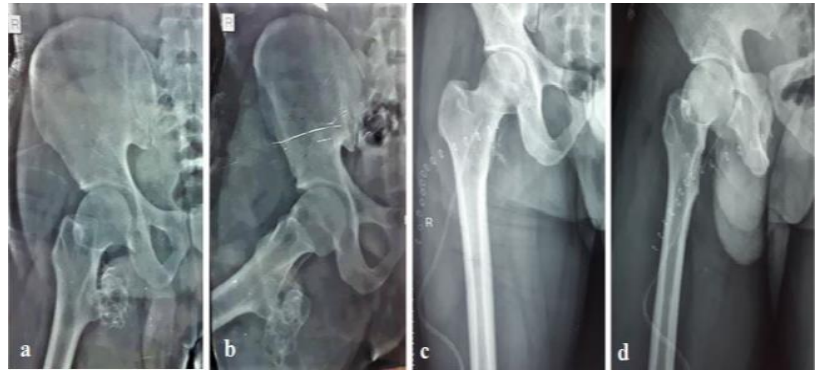

Figure 2: Radiograph of a 19 year old male showing

(a) and (b) a pedunculated osteochondroma of proximal femur, (c) and (d) the patient presented with restricted right hip movements, posterior approach to the hip was used to excise the tumor. Tumor removal can be seen on the post-operative radiograph.

\section{DISCUSSION}

This study included 32 patients with solitary osteochondromas of femur, who were managed with surgical excision of the tumor. In this series, 21 osteochondromas $(65.62 \%)$ were pedunculated and 11 osteochondromas $(34.37 \%)$ were sessile. This observation is similar to the one made by Khare in his series of 116 osteochondromas. ${ }^{14}$ In this study, $93.75 \%$ of the femoral osteochondromas were seen in the distal metaphysis and only $6.25 \%$ were seen in the proximal femur. This high occurrence in distal femoral metaphysis and rare involvement of the proximal femur in the present study is similar to the observations by Giudici et al. ${ }^{7}$ The present study involved 23 males $(71.87 \%)$ and nine females $(28.12 \%)$ with a male to female ratio of $2.5: 1$. This male preponderance is in affirmation with the previous literature. ${ }^{4,7}$ However, there is contrasting evidence available regarding the male preponderance of osteochondromas. Bottner et al in a series of 86 surgically managed symptomatic osteochondromas observed 46 males and 40 females; thus, nullifying any gender predilection. ${ }^{15}$ In the present study, the average age of the patients at the time of surgery was 17.5 years. The youngest patient operated was 11 years old and the oldest patient was 28 years old. Khare in his series of 116 osteochondromas observed a mean age of 13 years. ${ }^{14}$ Bottner et al in a series of 86 surgically managed symptomatic osteochondromas observed a mean age of 20 years. $^{15}$ In the present series, only the symptomatic osteochondromas were operated. The most common indication for surgery was pain, which was present in 18 patients $(56.25 \%)$. The other indications for surgery in the present study included cosmetic, fractured osteochondroma, restricted joint motion and paresthesia or numbness (Table 1). Giorgio et al in a series of 65 cases of surgically treated solitary osteochondromas also observed the pain to be the most common indication for surgery followed by nervous compression, esthetic injury and reduction in range of motion. ${ }^{16}$ In a series of 116 osteochondromas by Khare the indications for surgery included cosmetic causes, pain, joint motion hindrance, pressure on adjacent nerves, pathological fracture and 
malignant change. ${ }^{14}$ However, in the present study, no patient with malignant transformation, vascular compression or pathological fracture was observed. Few complications were encountered in the present series. The complications included post-operative hematoma formation, infection, post-operative hypoaesthesia and recurrence of the tumor. Post-operative hematoma was seen in two patients $(6.25 \%)$, both of which had giant osteochondromas of distal femur and were managed with serial aspiration and compressing bandages. Infection occurred in one case $(3.12 \%)$ which was treated with serial debribements and antibiotics. Hypoaesthesia in saphenous nerve distribution was seen in two patients $(6.25 \%)$ postoperatively which recovered within three months in both the patients. Recurrence was seen in one patient $(3.12 \%)$ in the present study. The recurrence occurred four years after primary surgery and was managed with re-surgery. Khare and Giorgio et al also observed similar trends with minimal complications which were dealt with easily. ${ }^{14,16}$

\section{CONCLUSION}

Pedunculated femoral osteochondromas are more common than the sessile ones. Distal metaphysis is the most common location for the femoral osteochondromas and the proximal femoral osteochondromas less common. Surgical excision of the symptomatic solitary femoral osteochondromas is a rewarding and safe procedure with minimal complications.

\section{ACKNOWLEDGEMENTS}

The authors acknowledge the contribution of all the faculty surgeons who performed the surgeries.

\section{Funding: No funding sources}

Conflict of interest: None declared

Ethical approval: The study was approved by the institutional ethics committee

\section{REFERENCES}

1. Khurana J, Abdul-Karim F, Bovée JVM. Osteochondroma. In: Fletcher CD, Unni KK, Mertens F (ed) Pathology and genetics of tumours of the soft tissues and bones. Lyon, IARC Press. 2002;234-7.

2. Lee KCY, Davies AM, Cassar-Pullicino VN. Imaging the complications of osteochondromas. Clin Radiol. 2002;57:18-28.

3. Bovee JVMG, Cleton-Jensen AM, Wuyts W. ExtMutation analysis and loss of heterozygosity in sporadic and hereditary osteochondromas and secondary chondrosarcomas. Am J Hum Genet. 1999;65:689-98.
4. Unni KK. Osteochondroma. In: Unni KK (ed) Dahlin's bone tumors: general aspects and data on 11,087 cases, 5th ed. Springfield: Thomas. 1996;1123.

5. Vanhoenacker FM, Van Hul W, Wuyts W, Willems PJ, De Schepper AM. Hereditary multiple exostoses: from genetics to clinical syndrome and complications. Eur J Radiol. 2001;40:208-17.

6. Murphey MD, Choi JJ, Kransdorf MJ, Flemming DJ, Gannon FH. Imaging of osteochondroma: variants and complications with radiologic-pathologic correlation. Radiographics. 2001;20(5):1407-34.

7. Giudici MA, Moser RP Jr, Kransdorf MJ. Cartilaginous bone tumors. Radiol Clin North Am. 1993;31(2):237-59.

8. Milgram JW. The origins of osteochondromas and enchondromas. A histopathologic study. Clin Orthop Relat Res. 1983;174:264-84.

9. Resnick D, Kyriakos M, Greenway GD. Osteochondroma. In: Resnick D (ed) Diagnosis of bone and joint disorders, 3rd edn. Philadelphia, Saunders. 1995;3725-46.

10. Scarborough MT, Moreau G. Benign cartilage tumors. Orthop Clin North Am. 1996;27(3):583-9.

11. Stieber JR, Dormans JP. Manifestations of hereditary multiple exostoses. J Am Acad Orthop Surg. 2005;13(2):110-20.

12. Lee JK, Yao L, Wirth CR. MR imaging of solitary osteochondromas: report of eight cases. AJR Am J Roentgenol. 1987;149(3):557-60.

13. de Souza AM, Bispo Júnior RZ. Osteochondroma: ignore or investigate? Rev Bras Ortop. 2014;49(6):555-64.

14. Khare GN. An analysis of indications for surgical excision and complications in 116 consecutive cases of osteochondroma. Musculoskelet Surg. 2011;95(2):121-5.

15. F Bottner, R Rodl, I Kordish, W Winklemann, G Gosheger, $N$ Lindner. Surgical treatment of symptomatic osteochondroma. A three- to eight-year follow-up study. J Bone Jt Surg Br. 2003;85(8):1161-5.

16. L Di Giorgio, R Lanzone, L Sodano, B Di Paola, G Touloupakis, M Mastantuono. Surgical treatment of osteochondromas: Indication in "Strategic exostosis". Clin Ter. 2015;166(1):27-33.

Cite this article as: Gupta S, Ghani A, Dev B, Mahajan R, Mohd J, Singh A, et al. Outcome analysis of solitary osteochondromas of femur managed surgically: a series of 32 patients. Int J Res Orthop 2021;7:82-5. 